\title{
Expression and Significance of GRP78 and HER-2 in Colorectal Cancer
}

\author{
Qiu-xia Ji¹, Li-li Wang², Lin Li³, Xiaoming Xing²
}

\author{
${ }^{1}$ Department of Pathology, Qingdao University, Qingdao, China \\ ${ }^{2}$ Department of Pathology, Affiliated Hospital of Qingdao University, Qingdao 266003, China \\ ${ }^{3}$ Department of Pathology, Yucheng People s Hospital, Yucheng, China
}

\begin{abstract}
Background and objective GRP78 and HER-2 expression are related to disease behaviors in various cancers. Previous research has shown that GRP78 is overexpressed in colorectal cancer and plays an important role in the development and progression of colorectal cancer. And evidence for HER-2 expression in colorectalcancer is contradictory for the protein expression status and prognostic value. In our study, we aim to investigate GRP78 and HER-2 expression in colorectal cancer and explore their association with clinicopathological features.

Methods Immnunohistochemical staining was used to assess the expression of GRP78 and HER-2 in 117 cases of colorectal carcinoma and corresponding adjacent tissues were selected for comparative analysis.

Results The positiverate of GRP78 and HER-2 were 68.4\% (80/117) and 38.5\% (45/117) respectively. The expression of GRP78 and HER-2 in gender, age, tumor size, tumor position, histological grade, depth of invasion and lymph node metastasis had no significant difference (all $P>0.05$ ); No relationship between GRP78 and HER-2 expression was detected $(P=0.089)$.

Conclusion GRP78 and HER-2 were not predictors for development and progression in CRC patients. The results do not support any relationship between GRP78 and HER-2 expression in this setting.
\end{abstract}

Keyword: GRP78, HER-2, Colorectal cancer, Immunohistochemistry

\begin{abstract}
Introduction
Colorectal cancer (CRC) is one of the most common gastrointestinal malignancies and still a major cause of cancer death in the world ${ }^{1}$. However, about $30-35 \%$ of patients would develop distant metastasis focus, with a median survival period of about 5 years 2. Although many factors have been reported to be related to the CRC occurrence, the molecular mechanisms which contribute to the pathogenesis and progression of CRC are still unclear. As a chief molecular chaperone in the endoplasmic reticulum (ER), glucose regulated protein (GRP78) is particular in modulating the unfolded protein response (UPR) ${ }^{3}$ and then maintaining the cell homeostasis. In addition, it can be translocated to the cell surface and serve as a receptor for one ligand to transmitting some signals for altering cell foundations ${ }^{4}$. However, GRP78 will become a protective protein when the tumor is formed. Recent studies have showed that GRP78 is over-expressed in CRC, and may play an important role in the development and progression of $\mathrm{CRC}^{5,6}$. HER-2 is a proto-oncogene that encodes a transmenbrane tyrosine kinas' receptor, the current
\end{abstract}

study has found that HER-2 is involved in the development, biological behavior and prognosis in various cancers. Siddiqa et al. ${ }^{7}$ demonstrated that HER-2 is overexpressed in 25-30\% of patients with breast cancer, and is correlated to the prognosis of breast cancer patients. However, the expression of HER-2 in CRC is contradictory ${ }^{2,8,9}$. In the present study, we carried an immunohistochemistry study to identify aberrantly expressed GRP78 and HER-2 in colorectal cancer and analyzed the correlations with the clinicopathological parameters.

\begin{abstract}
Materials and Methods
All CRC tissues were identified from The Affiliated Hospital of Qingdao University during a 3-year period between October 2010 and October 2013. The patients comprised 73 men and 44 women aged 25-85 years (mean:63.2y). All specimens were fixed in $10 \%$ formalin, embedded in paraffin, stained in $\mathrm{HE}$ and diagnosed by histology. None of the patients received anticancer treatment prior to surgery. Patients' clinicopathological parameters including gender, age, tumor differentiation, tumor size,
\end{abstract}

This article is published under the terms of the Creative Commons Attribution License 4.0 Author(s) retain the copyright of this article. Publication rights with Alkhaer Publications. Published at: http://www.ijsciences.com/pub/issue/2016-04/

DOI: 10.18483/ijSci.1001; Online ISSN: 2305-3925; Print ISSN: 2410-4477 
location, depth of invasion and lymph node metastasis.

\section{Immunohistochemistry}

Immunohistochemical staining was performed following the manufacturer's instructions. Paraffin sections $(4 \mu \mathrm{m}$ thick) were deparaffined in xylene, washed three times for 5 minutes each, then hydrated with an ethanol gradient, and then rinsed in water. After that, with $3 \% \mathrm{H}_{2} \mathrm{O}_{2}$ for $15 \mathrm{~min}$ to block endogenous peroxides activity. The slide were immersed in sodium citrated buffer (PH 6.0) with heating for 3 minutes for antigen retrieval. And then the slides were incubated with the rabbit anti-GRP78 polyclone antibody (1:250, Abcam, USA) and anti-rabbit HER-2 monoclone antibody (1:100, Roche, USA) overnight at $4^{\circ} \mathrm{C}$ temperature. After that, the slides were incubated with the peroxides-conjugated anti-rabbit antibody (Roche, USA) for $30 \mathrm{~min}$ at room temperature, diaminobenzidine (DAB) was used as the chromate agent and counterstained with hematoxylin. Negative controls were performed by replacing the primary antibodies with PBS. The breast cancer specimen was used as positive control according to manufacturer's instructions. DAB and PBS were purchased from Roche.

\section{Immunohistochemistry evaluation}

Two high qualification pathologist determined the final scores under optics microscope by consensus. The expression of GRP78 was located in cytoplasm and membrane. GRP78 staining was scored semi-quantitatively according to the manufacturer's guidelines. Score0: no staining; score $1+: \leq 30 \%$ of tumor cells expressing; score $2+$ : between $31 \%$ and $50 \%$ of tumor cells expressing; score $3+$ : $>51 \%$ of tumor cells expressing. Scores of 0 and $1+$ were classified as negative and scores of $2+$ and $3+$ were classified as positive. HER-2 staining was scored semi quantitatively according to the manufacturer's instructions: 0: no staining or membrane staining < $10 \%$ of the tumor cells; $1+$ : week membrane staining or $\geq 10 \%$ of tumor cells expressing; $2+$ : weak-to-moderately complete membrane staining of $\geq 10 \%$ of tumor cells; $3+$ : a strongly complete membrane staining of $\geq 10 \%$ of tumor cells. Score of 0 indicated negative for HER-2 expression, 1+, 2+ and $3+$ were regarded as positive expression of HER-2。

\section{Statistics analyses}

The results were analyzed using SPSS19.0 (SPSS, USA).Association between expression of the two proteins and patient clinicopathological parameters were analyzed by the Chi-Squared test or Fisher's exact test. $P$ values $<0.05$ were considered statistically significant.

\section{Results \\ The expression of GRP78 and HER-2 expression in colorectal cancer}

GRP78 and HER-2 did not expressed in adjacent tissues. GRP78 expression were positive in 80 of 117 colorectal cancer samples $(68.4 \%)$, the pattern of GRP78 immunostaining was both membranous and cytoplasm (Fig.1A). Of 117 cases examined, 45 (38.5\%) was HER-2 positive (Fig.1B), HER-2 was expressed in membranous.

Correlation of GRP78, HER-2expression and clinicopathological features

Expression of GRP78 and HER-2 in CRC with clinical features was shown in Table 1. Statistical evaluation of GRP78 and HER-2 expression according to gender, age, tumor location, tumor size, differentiation grade, depth of invasion or lymph node metastasis revealed no significant difference among these variables (all $P>0.05$ ).

\section{The association between GRP78 and HER-2 expression}

There was no significant positive correlation between GRP78 and HER-2 expression in CRC ( $\mathrm{r}=0.122$; $P>0.05)$ (Table 2).

\section{Discussion}

GRP78, a crucial protein that is located in ER and belongs to HSP70 protein family, can be induced by the ER stress response ${ }^{10}$. It is widely accepted that the over-expression of GRP78 plays an important roles in the process of CRC, including: transfer the unfold or misfiled proteins, maintain protein synthesis under the circumstance of ER stress, stabilize intracellular calcium homeostasis ${ }^{11}$. In the development of CRC, ER stress response, cell repair and apoptosis are important process of pathophysiology.

In the present study, for 117 CRC samples, $68.4 \%$ (80/117) showed positive expression of GRP78. Same result was described in our previous study ${ }^{5,6}$. In previous study, GRP78 expression was elevated gradually in human normal colorectal tissues, adenoma specimens and colorectal carcinoma specimens. In addition, we found that GRP78 is overexpressed in CRC patients but not associated with clinicopathological parameters. It demonstrated that GRP78 may participate in the development and progression of colorectal neoplasm, although with no significant of prognosis.

HER-2 is a member of tyrosine kinas' receptor super family and homologous to epidermal growth factor receptor (EGFR). It is referred to the growth and progression of malignant cells. The expression of HER-2 in CRC was not consistent with reported ${ }^{8,9,12}$ as well as the relationship between tumors' 
development and progression. Kapitanovi et.al. ${ }^{9}$ demonstrated that normal mucosa was mostly negative, but significant number of benign lesions and CRC overexpressed HER-2. Adenocarcinomas were significantly more positive than benign. The expression level of HER-2 can be used as an independent prognostic factor for CRC patients. Kovaeevi et $\mathrm{al}^{13}$ demonstrated that this oncoprotein was predictive neither for overall survival nor for invasion and metastases in rectal cancer patients. Our data showed that HER-2 overexpressed in $38.5 \%$ of cases while this did not correlate with clinicopathological parameters, similar to the study by Schuel et al. ${ }^{14}$. All of these above indicated that overexpression of HER-2 plays an important role in the progression of CRC and is not considered as an independent indicator for invasion and prognostic of CRC.

Previous report ${ }^{15}$ has revealed that GRP78-EGFR complex can inhibited the EGFR singling pathway, while EGFR and HER-2 are similar in structure and function. Several studies show that the two proteins co-expression in colorectal cancer, the synergistic effect existed in the tumor genesis and biological behavior $^{16,17}$, so that there will be a correlation between GRP78 and HER-2. However, our findings suggest that there is no apparent correlation between GRP78 and HER-2 expression in CRC. Therefore, they may no correlation between GRP78 and HER-2 expression, specific regulatory mechanism between the two has no relevant research reports.

Our study aimed to evaluate the expression of GRP78 and HER-2 in CRC by IHC methods. We indicated that both GRP78 and HER-2 were not predictors for development and progression in CRC patients, and our results do not support an association between GRP78 and HER2 expression.

\section{Acknowledgements}

This work was supported by the grants from the National Natural Science Foundation of China (No.81201947) .

\section{References}

1) 1. Ferlay J, Soerjomataram I, Dikshit R, et al. Cancer incidence and mortality worldwide: sources, methods and major patterns in GLOBOCAN 2012. International journal of cancer. Mar 1 2015;136(5):E359-386.

2) 2. Ingold Heppner B, Behrens HM, Balschun K, et al. HER2/neu testing in primary colorectal carcinoma. British journal of cancer. Nov 11 2014;111(10):1977-1984.
3) 3. $\mathrm{Ni} \mathrm{M}$, Lee AS. ER chaperones in mammalian development and human diseases. FEBS letters. Jul 31 2007;581(19):3641-3651.

4) 4. Li Z, Zhang L, Li H, Shan S, Li Z. Glucose regulated protein 78 promotes cell invasion via regulation of uPA production and secretion in colon cancer cells. BMB reports. Aug 2014;47(8):445-450.

5) 5. Xing X, Li Y, Liu H, Wang L, Sun L. Glucose regulated protein 78 (GRP78) is overexpressed in colorectal carcinoma and regulates colorectal carcinoma cell growth and apoptosis. Acta histochemica. Dec 2011;113(8):777-782.

6) 6. Xing $\mathrm{X}$, Lai $\mathrm{M}$, Wang $\mathrm{Y}$, $\mathrm{Xu} \mathrm{E}$, Huang $\mathrm{Q}$. Overexpression of glucose-regulated protein 78 in colon cancer. Clinica chimica acta; international journal of clinical chemistry. Feb 2006;364(1-2):308-315.

7) 7. Siddiqa A, Long LM, Li L, Marciniak RA, Kazhdan I. Expression of HER-2 in MCF-7 breast cancer cells modulates anti-apoptotic proteins Survivin and Bcl-2 via the extracellular signal-related kinase (ERK) and phosphoinositide-3 kinase (PI3K) signalling pathways. BMC cancer. 2008;8:129.

8) 8. Marx AH, Burandt EC, Choschzick M, et al. Heterogenous high-level HER-2 amplification in a small subset of colorectal cancers. Human pathology. Nov 2010;41(11):1577-1585.

9) 9. Kapitanovic S, Radosevic S, Kapitanovic M, et al. The expression of p185(HER-2/neu) correlates with the stage of disease and survival in colorectal cancer. Gastroenterology. Apr 1997;112(4):1103-1113.

10) 10. Li Z, Li Z. Glucose regulated protein 78: a critical link between tumor microenvironment and cancer hallmarks. Biochimica et biophysica acta. Aug 2012;1826(1):13-22.

11) 11. Pfaffenbach KT, Lee AS. The critical role of GRP78 in physiologic and pathologic stress. Current opinion in cell biology. Apr 2011;23(2):150-156.

12) 12. Yao YF, Du CZ, Chen N, Chen P, Gu J. Expression of HER-2 in rectal cancers treated with preoperative radiotherapy: a potential biomarker predictive of metastasis. Diseases of the colon and rectum. May 2014;57(5):602-607.

13) 13. Kovacevic D, Sonicki Z, Kusic Z, et al. Preoperative serum levels of c-erbB-2 do not seem to be useful in management of patients with rectal cancer. International journal of colorectal disease. Jul 2007;22(7):827-831. 
14) 14. Schuell B, Gruenberger T, Scheithauer W, Zielinski C, Wrba F. HER 2/neu protein expression in colorectal cancer. BMC cancer. 2006;6:123.

15) 15. Cai B, Tomida A, Mikami K, Nagata K, Tsuruo T. Down-regulation of epidermal growth factor receptor-signaling pathway by binding of GRP78/BiP to the receptor under glucose-starved stress conditions. Journal of cellular physiology. Nov 1998;177(2):282-288.
16) 16. Kavanagh DO, Chambers G, O'Grady L, et al. Is overexpression of HER-2 a predictor of prognosis in colorectal cancer? BMC cancer. 2009;9:1

17) 17. Ma WW, Adjei AA. Novel agents on the horizon for cancer therapy. CA: a cancer journal for clinicians. Mar-Apr 2009;59(2):111-137.

Table 1 Clinicopathological variables and their correlation with immunohistochemical expression of GRP78 and HER-2

\begin{tabular}{|c|c|c|c|c|c|c|c|}
\hline \multirow{2}{*}{ Variable } & \multirow{2}{*}{ Total } & \multicolumn{2}{|c|}{ GRP78 } & \multirow{2}{*}{$P$-Value ${ }^{a}$} & \multicolumn{2}{|c|}{ HER-2 } & \multirow{2}{*}{$P$-Value ${ }^{a}$} \\
\hline & & - & + & & - & + & \\
\hline \multicolumn{8}{|l|}{ Gender } \\
\hline Male & 73 & 26 & 47 & \multirow{2}{*}{0.232} & 47 & 26 & \multirow{2}{*}{0.415} \\
\hline Female & 44 & 11 & 33 & & 25 & 19 & \\
\hline \multicolumn{8}{|l|}{ Age(year) } \\
\hline$>55$ & 24 & 8 & 16 & \multirow{2}{*}{0.840} & 13 & 11 & \multirow{2}{*}{0.405} \\
\hline$\leq 55$ & 93 & 29 & 64 & & 59 & 34 & \\
\hline \multicolumn{8}{|l|}{ Tumor site } \\
\hline Colon & 54 & 17 & 37 & \multirow{2}{*}{0.976} & 36 & 18 & \multirow{2}{*}{0.291} \\
\hline Rectal & 63 & 20 & 43 & & 36 & 27 & \\
\hline \multicolumn{8}{|l|}{ Tumor size $(\mathrm{cm})$} \\
\hline$>3$ & 96 & 28 & 68 & \multirow{2}{*}{0.222} & 58 & 38 & \multirow{2}{*}{0.594} \\
\hline$\leq 3$ & 21 & 9 & 12 & & 14 & 7 & \\
\hline \multicolumn{8}{|l|}{ Differentiation grade } \\
\hline Well and Moderate & 78 & 23 & 55 & \multirow{2}{*}{0.482} & 51 & 27 & \multirow{2}{*}{0.227} \\
\hline Poor & 39 & 14 & 25 & & 21 & 18 & \\
\hline \multicolumn{8}{|l|}{ Serosal invasion } \\
\hline No & 8 & 2 & 6 & \multirow{2}{*}{$1.000^{\mathrm{b}}$} & 5 & 3 & \multirow{2}{*}{$1.000^{\mathrm{b}}$} \\
\hline Yes & 109 & 35 & 74 & & 67 & 42 & \\
\hline \multicolumn{8}{|l|}{$\begin{array}{l}\text { Lymph node } \\
\text { metastasis }\end{array}$} \\
\hline Positive & 84 & 23 & 61 & \multirow{2}{*}{0.115} & 49 & 35 & \multirow{2}{*}{0.256} \\
\hline Negative & 33 & 14 & 19 & & 23 & 10 & \\
\hline
\end{tabular}

a Pearson $x^{2}$

test

${ }^{\mathrm{b}}$ Adjusted $\mathrm{x}^{2}$ test 
Table 2 Relation between GRP78 and HER-2 expression

\begin{tabular}{ccccccc}
\hline GRP78 & Total & \multicolumn{2}{c}{ HER-2 expression } & & r & $P$-value \\
\cline { 3 - 4 } expression & & + & - & & 0.122 & 0.089 \\
\hline+ & 80 & 34 & 46 & & 0.122 \\
\hline
\end{tabular}
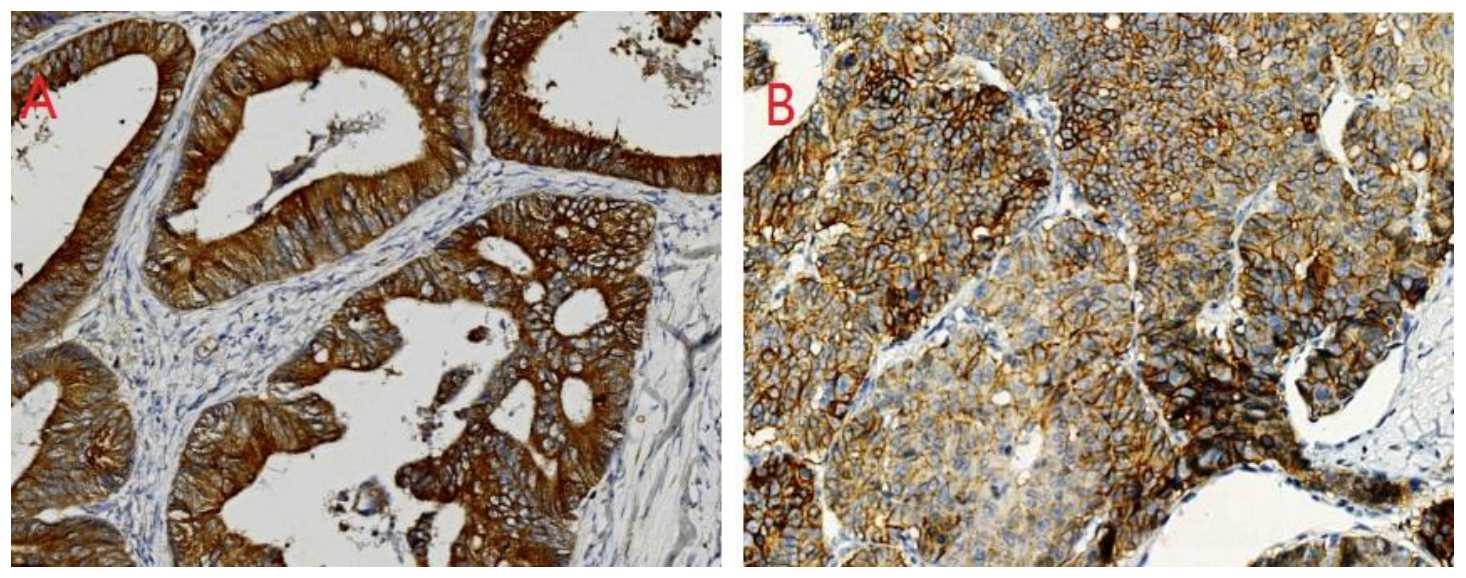

Fig1 Immunohistochemical staining of GRP78 and HER-2 in colorectal cancer. A:GRP78-positive expression in colorectal cancer, magnification $\times 400$; B: HER-2 positive expression in colorectal cancer, magnification $\times 400$ 